\title{
ESQUIZO-Q: Un Instrumento para la Valoración del "Alto Riesgo Psicométrico" a la Psicosis
}

\section{ESQUIZO-Q: An Instrument for the Assessment of "Psychometric High Risk" for Psychosis}

\author{
Eduardo Fonseca-Pedrero \\ Universidad de Oviedo y CIBERSAM \\ Oscar Vallina-Fernández \\ Hospital de Sierrallana, Torrelavega, Santander \\ Mercedes Paino \\ Universidad de Oviedo y CIBERSAM
}

\author{
Serafín Lemos-Giráldez \\ Universidad de Oviedo y CIBERSAM \\ José Muñiz \\ Universidad de Oviedo y CIBERSAM
}

\begin{abstract}
Resumen. El paradigma de "alto riesgo psicométrico" tiene como finalidad la detección, mediante la utilización de autoinformes y a partir de su perfil de puntuaciones, de aquellos participantes con una mayor vulnerabilidad teórica de transitar hacia un trastorno psicótico en el futuro. La detección temprana de este tipo de individuos de riesgo pasa por disponer de adecuados instrumentos de evaluación que nos permitan tomar decisiones sólidas y fundadas a partir de sus puntuaciones. El principal objetivo de este estudio fue presentar el proceso de construcción y validación de un autoinforme de reciente creación denominado ESQUIZO-Q: Cuestionario Oviedo para la Evaluación de la Esquizotipia. El análisis de la estructura interna arrojó una solución tridimensional: Distorsión de la Realidad, Anhedonia y Desorganización Interpersonal. Los niveles de consistencia interna para las subescalas del ESQUIZO-Q oscilaron entre 0,62 y 0,90. Ningún ítem presentó un funcionamiento diferencial en función del sexo de los participantes. Asimismo, las subescalas del ESQUIZO-Q correlacionaron de forma moderada con las escalas de otros autoinformes que valoraban depresión, problemas comportamentales y rasgos de los trastornos de la personalidad. El ESQUIZO-Q es un instrumento de medida breve, sencillo y con adecuadas propiedades psicométricas, que puede ser utilizado como herramienta epidemiológica y como método de screening psicopatológico en población general adolescente. Futuras investigaciones deberían realizar estudios longitudinales con la finalidad de examinar la capacidad predictiva del ESQUIZO-Q.

Palabras clave: esquizotipia, propensión a la psicosis, autoinforme, riesgo, esquizotípica.
\end{abstract}

\begin{abstract}
The aim of the "psychometric high risk" paradigm is the detection, by means of self-report measures and based on score profiles, of those individuals with a higher theoretical vulnerability of moving toward a psychotic disorder in the future. The early detection of this type of at-risk individual requires adequate assessment instruments that allow us to make solid and well-founded decisions based on their scores. The main objective of this study was to present the construction and validation of a recently created self-report called ESQUIZO-Q: Cuestionario Oviedo para la Evaluación de la Esquizotipia (The Oviedo Schizotypy Assessment Questionnaire). The analysis of its internal structure revealed a three-factor solution: Distortion of Reality, Anhedonia and Interpersonal Disorganization. The levels of internal consistency for the ESQUIZO-Q subscales ranged from 0.62 to 0.90 .
\end{abstract}

La correspondencia sobre este artículo debe enviarse al primer autor a la Universidad de Oviedo, Facultad de Psicología, Plaza Feijoo, s/n, 33003, Oviedo, España. e-mail: efonseca@cop.es
Agradecimientos: Esta investigación ha sido financiada por el Ministerio de Ciencia e Innovación (MICINN) de España (BES-200612797, PSI 2008-06220, PSI 2008-03934) y por el Instituto Carlos III, Centro de Investigación Biomédica en Red de Salud Mental (CIBER$S A M)$. 
No item showed differential functioning as a function of the gender of participants. Likewise, the ESQUIZO-Q subscales correlated moderately with the scales of other selfreports that assess depression, behavioral problems and personality disorder traits. The ESQUIZO-Q is a brief and easy self-report instrument with adequate psychometric properties that can be used as an epidemiological tool and as a psychopathological screening method in the general population. Future investigations should conduct longitudinal studies in order to examine the predictive capacity of the ESQUIZO-Q.

Key words: schizotypy, psychosis propensity, self-report, risk; schizotypal.

\section{Hacia una detección e intervención temprana de la esquizofrenia}

La esquizofrenia es un trastorno mental devastador que impacta directamente en las esferas personal, familiar y social del individuo, afectando aproximadamente al $1 \%$ de la población, y que tiene su inicio en la adolescencia tardía y/o temprana adultez (American Psychiatric Association, 2000; van Os y Kapur, 2009). Es por ello que la prevención y detección temprana de este trastorno constituye una de las principales prioridades en materia de salud mental de las autoridades sanitarias. La evidencia clínica sugiere que, una rápida detección del cuadro psicótico, con una posterior intervención temprana eficaz, no sólo mejora el pronóstico del trastorno a corto, medio y largo plazo, sino que también incidirá en una mejora de los resultados del tratamiento (Lemos-Giráldez et al., 2009; McGlashan y Johannessen, 1996; Yung, Philips, Pan Yuen y McGorry, 2004). Más aún, en caso de aparición del trastorno, la recuperación será mucho más rápida, disminuyendo el tiempo de hospitalización y las consecuencias negativas asociadas al cuadro clínico (p. ej., comorbilidad) y al impacto que ocasiona el trastorno (McGorry, Krstev y Harrigan, 2000; Vallina, Lemos Giráldez y Fernández, 2006). Los costes sanitarios, familiares y sociales que se derivan de la esquizofrenia (p. ej., psicofármacos, hospitalización, seguimiento en la comunidad) son muy elevados, por lo que todas las acciones que se lleven a cabo para prevenir este problema son de un enorme interés y utilidad.

Las estrategias de prevención se pueden dividir en tres tipos: universales, selectivas e indicadas (Brown y Faraone, 2004; Yung et al., 2007). La prevención universal va directamente encaminada a la población general; un claro ejemplo serían las cam- pañas de tráfico y seguridad vial, o las vacunaciones en epidemias que potencialmente afectan a toda la población. Por su parte, las intervenciones selectivas tienen como meta a aquellas personas con mayor riesgo de padecer alguna enfermedad física o algún problema psicológico, y que sin embargo no muestran ningún signo o síntoma de enfermedad física o de trastorno psicológico evidente (p. ej., hijos de padres con problemas psicológicos o enfermedades con carga genética demostrada). La tercera aproximación, la prevención indicada, se centra en detectar a aquellos individuos con vulnerabilidad que ya muestran ciertos signos y síntomas similares a los problemas psicológicos señalados en los manuales diagnósticos al uso (American Psychiatric Association, 2000), pero que aún no alcanzan niveles clínicos que permitan diagnosticarlos formalmente. Las intervenciones universal y selectiva conllevan un enorme gasto económico y material, por lo que en el campo de los trastornos mentales lo más aconsejable sería la implementación de programas de intervención indicada. Concretamente, en el campo de la esquizofrenia y sus trastornos relacionados, existe un método de investigación focalizado en la detección, mediante autoinformes, de participantes que informan de experiencias psicóticas atenuadas similares a las encontradas en pacientes con esquizofrenia: es el denominado paradigma de "alto riesgo psicométrico".

\section{Estudios de "alto riesgo psicométrico": ¿Es posible una detección temprana de individuos de riesgo de esquizofrenia mediante autoinformes?}

La idea de prevenir e intervenir precozmente en personas con riesgo de padecer esquizofrenia, con la finalidad de mitigar su posible impacto a múltipes 
niveles, ha impulsado el desarrollo y la validación de una amplia variedad de instrumentos de medida para la valoración de la esquizotipia o, más genéricamente, de la propensión a la psicosis (FonsecaPedrero et al., 2008). Esta línea de investigación centrada en el uso de cuestionarios -o la obtención de un perfil de puntuaciones-, cuya finalidad es identificar a aquellos participantes con mayor probabilidad de desarrollar trastornos del espectro esquizofrénico en el futuro, se conoce en la literatura como paradigma de "alto riesgo psicométrico" (Lenzenweger, 1994). En la actualidad, este método de investigación es considerado como una estrategia fiable, válida y útil para la detección psicométrica de individuos con vulnerabilidad a la esquizofrenia, de cara a la posible implementación posterior de tratamientos profilácticos. Del mismo modo, la utilización de estos instrumentos de medida constituye, en comparación con otras técnicas, un sistema de evaluación rápido, eficaz y no invasivo (Gooding, Tallent y Matts, 2005; Kwapil, Barrantes Vidal y Silvia, 2008), que soslaya los efectos de confundido frecuentemente asociados a los pacientes con esquizofrenia, como los derivados de la medicación o la estigmatización. Más aún, los estudios de "alto riesgo psicométrico" se pueden utilizar en combinación con otras estrategias de investigación, tales como el paradigma de alto riesgo genético (evaluación de familiares de primer grado de pacientes con esquizofrenia), con la finalidad de analizar los posibles mecanismos etiopatogénicos involucrados en este tipo de trastornos (Kwapil et al., 2008).

La piedra angular de este paradigma de investigación se fundamenta en los datos procedentes de los análisis de validez predictiva. Estudios longitudinales independientes indican que los individuos de la población general que informan de experiencias esquizotípicas, tales como pensamiento mágico, experiencias alucinatorias, ideación delirante y/o anhedonia (incapacidad para experimentar placer), tienen un mayor riesgo futuro de transitar hacia un trastorno del espectro esquizofrénico en comparación con aquellos que no presentan tales síntomas y signos (Chapman, Chapman, Raulin y Eckblad, 1994; Dominguez, Wichers, Lieb, Wittchen y van Os, in press; Gooding et al., 2005; Kwapil, 1998; Poulton et al., 2000; Welham et al., 2009).
Resultados similares se encuentran cuando se analiza la presencia de este tipo de experiencias en familiares de primer grado de pacientes con esquizofrenia (Johnstone, Ebmeier, Miller, Owen y Lawrie, 2005), así como en participantes con pródromos -síntomas previos a la aparición del episodio psicótico- (Morrison et al., 2006). En este sentido, se podrían considerar las experiencias esquizotípicas como un marcador de riesgo exofenotípico para la esquizofrenia (Raine, 2006) o como la expresión conductual de vulnerabilidad a la psicosis (van Os, Linscott, Myin-Germeys, Delespaul y Krabbendam, 2009).

Los individuos que refieren experiencias esquizotípicas también presentan un mayor grado de alteración afectiva, cognitiva, social, interpersonal y comportamental (Barrantes Vidal, Ros-Morente y Kwapil, 2009; Fonseca-Pedrero, Lemos-Giráldez, Paíno-Piñeiro, Villazón-García y Muñiz, 2010; Kwapil et al., 2008; Lenzenweger, McLachlan y Rubin, 2007; Raine, 2006), claro indicativo de su estado mental de riesgo. Estos datos parecen indicar que las alteraciones características de los pacientes con esquizofrenia se pueden encontrar también en muestras de la población general por debajo del umbral clínico, apoyando la hipótesis de la continuidad del fenotipo psicótico (van Os et al., 2009). De acuerdo con esta hipótesis, las experiencias esquizotípicas se situarían en algún punto de este continuo, y podrían verse como un fenotipo "intermedio", cualitativamente y fenomenológicamente similar, pero cuantitativamente menos grave, que la sintomatología encontrada en pacientes con esquizofrenia, presentándose con una menor intensidad, persistencia, frecuencia y discapacidad asociada (Dominguez et al., in press; Fonseca-Pedrero, Lemos-Giráldez, Paino et al., 2010; Obiols, Barragán, Vicens y Navarro, 2008). Más aun, la gran mayoría de los individuos que informan de experiencias esquizotípicas se muestran clínicamente compensados y no desarrollarán nunca un cuadro psicótico; no obstante, en un subgrupo determinado de individuos, la confluencia de otros factores genéticos (p. ej., familiares de pacientes con esquizofrenia) y/o ambientales (p. ej., traumas psicológicos, consumo de cannabis o sintomatología depresiva), podría aumentar la persistencia y la estabilidad tem- 
poral de estas experiencias, incrementando de este modo el riesgo de psicosis y la necesidad de tratamiento (van Os et al., 2009).

\section{Instrumentos de medida disponibles para la valoración de la esquizotipia}

En la literatura se pueden hallar diferentes instrumentos de evaluación para la valoración de la esquizotipia tanto en población adulta como en adolescentes. En adultos existen diversos autoinformes, entre ellos, el Schizotypal Personality Questionnaire (SPQ) (Raine, 1991), las escalas Wisconsin de propensión a la psicosis (Chapman, Chapman y Kwapil, 1995) o el Oxford-Liverpool Feelings and Experiences (O-LIFE) (Mason y Claridge, 2006). Asimismo, y dado que la adolescencia es un periodo evolutivo de especial riesgo para el desarrollo de trastornos del espectro esquizofrénico, también se han realizado esfuerzos para la valoración de la propensión a la psicosis en este sector de la población; dos claros ejemplos de ello son las Junior Schizotypy Scales (JSS) (Rawlings y MacFarlane, 1994) y el Schizotypy Traits Questionnaire (STA) for children (Cyhlarova y Claridge, 2005). Estos autoinformes, aunque son pioneros en este campo de estudio, presentan algunas limitaciones. Por ejemplo, en el caso del JSS, se han encontrado niveles de consistencia interna cercanos a 0,50, así como una solución factorial inestable (DiDuca y Joseph, 1999; Rawlings y MacFarlane, 1994), hecho que ha impulsado el desarrollo de una versión reducida (FonsecaPedrero, Linscott, Lemos-Giráldez, Paino y Muñiz, in press). Por su parte, el STA for children, en su validación, presentó un correcto comportamiento psicométrico; sin embargo, como mencionan Cyhlarova y Claridge (2005), este autoinforme no considera la dimensión Anhedonia de la esquizotipia, la cual ha demostrado ser relevante en la predicción del riesgo de los trastornos del espectro esquizofrénico (Gooding et al., 2005; Kwapil, 1998). Estos aspectos se unen a la necesidad de la incorporación de los avances en la medición psicológica y educativa, como por ejemplo, la construcción de tests e ítems mediante un proceso estandarizado, la introducción del formato de respuesta tipo Likert, el análisis del funcionamiento diferencial de los ítems, o el desarrollo de baremos específicos en muestras representativas de la población general adolescente. Por otra parte, hasta el momento no existía un instrumento específicamente diseñado para la valoración de los rasgos esquizotípicos en población adolescente española, todo lo cual justifica la construcción del ESQUIZO-Q: Cuestionario Oviedo para la Evaluación de la Esquizotipia (FonsecaPedrero, Muñiz, Lemos-Giráldez, Paino y VillazónGarcía, 2010). La disponibilidad de instrumentos de medida breves, sencillos y de rápida aplicación, que puedan ser utilizados como método de cribado para la detección de participantes de riesgo a los trastornos del espectro esquizofrénico, de cara a una evaluación psicológica más exhaustiva, es de suma importancia, tanto desde un punto de vista clínico como investigador.

\section{Proceso de construcción y validación del ESQUIZO-Q}

\section{a) Construcción del ESQUIZO-Q}

El proceso de construcción del ESQUIZO-Q (Fonseca-Pedrero, Muñiz et al., 2010) fue definido objetivamente siguiendo las directrices internacionales para la construcción de instrumentos de medida (American Educational Research Association, American Psychological Association y National Council on Measurement in Education, 1999; Downing, 2006; Muñiz y Fonseca-Pedrero, 2008), y de acuerdo a unos principios teóricos y métricos que permitiesen maximizar su validez y, por lo tanto, las inferencias y decisiones que se fuesen a derivar de las puntuaciones de los participantes (Downing, 2006).

El ESQUIZO-Q es un autoinforme creado para evaluar los rasgos esquizotípicos de la personalidad en población adolescente, el cual está fundamentado en los criterios diagnósticos propuestos para este trastorno en el DSM-IV-TR (American Psychiatric Association, 2000) y en el modelo de esquizotaxia de Meehl (1962) sobre predisposición genética a la esquizofrenia. Los ítems del ESQUIZO-Q fueron seleccionados a partir de una revisión exhaustiva de 
la literatura previa (Fonseca-Pedrero et al., 2008), considerando un total de 10 facetas, a saber: Ideación Referencial, Pensamiento Mágico, Experiencias Perceptivas Extrañas, Pensamiento y Lenguaje Extraños, Ideación Paranoide, Anhedonia Física, Anhedonia Social, Comportamiento Raro, Falta de Amigos Íntimos y Ansiedad Social Excesiva. El formato de respuesta utilizado fue tipo Likert de 5 categorías (desde 1 "Completamente en desacuerdo" a 5 "Completamente de acuerdo"). En la elaboración de los ítems también se tuvieron en cuenta las directrices internacionales propuestas para la construcción de ítems (Moreno, Martínez y Muñiz, 2006; Moreno, Martínez y Muñiz, 2004; Muñiz, Fidalgo, García-Cueto, Martínez y Moreno, 2005). Además, algunos ítems se formularon en términos positivos y otros en términos negativos para evitar la aquiescencia. En un primer momento, dado que el objetivo final era crear un cuestionario breve en torno a 50 ítems, y según las recomendaciones psicométricas de construir como mínimo el doble de ítems, se partió de un banco inicial de 141 ítems.

Para la construcción y validación del cuestionario, se llevaron a cabo dos estudios piloto (cualitativo y cuantitativo) y un estudio final de campo. En el estudio piloto cuantitativo, participaron un total de 306 adolescentes $(M=15,7$ años; $D T=1,3)$ a los que se les administró el conjunto inicial de 141 ítems. Este conjunto preliminar de ítems se redujo a 110 en función de los criterios psicométricos de depuración que se mencionan a continuación: 1) Ítems que presentaban índices de discriminación inferiores a 0,$30 ; 2$ ) Ítems que no se agruparan en una estructura esencialmente unidimensional; 3) Ítems con cargas factoriales inferiores a 0,30 (una vez analizada la unidimensionalidad); y 4) Ítems en los que se les detectó funcionamiento diferencial en función del sexo. Este segundo bloque de 110 ítems se administró, junto con otros autoinformes, en el estudio de campo a una muestra de 1683 estudiantes $(M=15,9$ años; $D T=1,2)$, seleccionados mediante un muestro estratificado por conglomerados a nivel de aula en el Principado de Asturias (España). Participaron un total de 41 centros educativos y 91 aulas. En el estudio también se aplicó la Escala Oviedo de
Infrecuencia de Respuesta (Fonseca-Pedrero, Lemos-Giráldez, Paino, Villazón-García y Muñiz, 2009), con la finalidad de detectar a los participantes que pudiesen responder de forma azarosa o deshonesta. En función de los criterios psicométricos de reducción de ítems mencionados, el ESQUIZO-Q quedó finalmente formado por un total de 51 ítems distribuidos a lo largo de 10 subescalas. Las propiedades psicométricas del mismo se mencionan a continuación, si bien para un análisis más pormenorizado de las mismas se puede consultar el manual publicado del cuestionario (Fonseca-Pedrero, Muñiz et al., 2010).

\section{b) Obtención de evidencias de validez de las puntuaciones del ESQUIZO-Q}

\section{Evidencias de validez de contenido}

La validez de contenido se examinó a partir del juicio de expertos y de una revisión exhaustiva de los diferentes autoinformes existentes en la literatura para la evaluación de la esquizotipia y de otros constructos relacionados con ella. En la elaboración y revisión de los ítems del ESQUIZO-Q participaron tres expertos en la construcción de cuestionarios, y tres expertos en el campo de la esquizofrenia. Los bancos de ítems administrados, tanto en el estudio piloto como en el estudio final de campo, fueron revisados mediante un proceso iterativo, y recibieron la aprobación del grupo de expertos.

\section{Estructura dimensional del ESQUIZO-Q}

Con la finalidad de obtener evidencias de validez acerca de la estructura interna del ESQUIZOQ, se llevó a cabo un Análisis de componentes principales (ACP) de primer y segundo orden. El ACP de primer orden arrojó una estructura factorial formada por un total de 11 componentes que replicaba fidedignamente las subescalas hipotetizadas por los constructores del cuestionario, a excepción de la faceta Falta de Amigos Íntimos que se disgregó en dos (relaciones con los familiares y relación con los amigos). Con los componentes de 
primer orden se realizó un ACP de segundo orden que arrojó una solución tridimensional, concretada en las dimensiones: Distorsión de la realidad (Experiencias Percep-tivas Extrañas, Ideación Paranoide, Pensamiento Mágico e Ideación Referencial), Anhedonia (Anhedonia Física y Anhedonia Social) y Desorganización Interpersonal (Ansiedad Social Excesiva, Falta de Amigos Íntimos I y II, Comportamiento Raro y Pensamiento y Lenguaje Extraños). Los resultados del estudio de la dimensionalidad subyacente a los ítems del ESQUIZO-Q se recogen en la Tabla 1.

Tabla 1. Análisis de componentes principales de segundo orden del ESQUIZO-Q: Cuestionario Oviedo para la Evaluación de la Esquizotipia

\begin{tabular}{lccr}
\hline & \multicolumn{3}{c}{ Componentes de segundo orden } \\
\hline Componentes de primer orden & I & II & III \\
\hline Pensamiento Mágico &, 76 & & \\
Experiencias Perceptivas Extrañas &, 74 & & \\
Ideación Referencial &, 62 & & \\
Ideación Paranoide &,- 47 &,- 37 & \\
Falta de Amigos Íntimos I & &, 63 & \\
Comportamiento Raro & &,- 54 & \\
Falta de Amigos Íntimos II & &,- 53 & \\
Ansiedad Social Excesiva & &, 51 & \\
Pensamiento y Lenguaje Raros & &, 49 & \\
Anhedonia Física & & &,- 79 \\
Anhedonia Social & & &, 77 \\
Autovalor & & 1,30 & 1,16 \\
\% Varianza explicada & 2,46 & 11,84 & 10,56 \\
\% Varianza explicada acumulada & 22,38 & 34,22 & 44,79 \\
\hline
\end{tabular}

Nota: se han eliminado las cargas factoriales inferiores a , 35

\section{Evidencias de validez convergente}

Con la finalidad de obtener fuentes de validez convergente de las puntuaciones del ESQUIZO-Q, se examinaron las correlaciones de Pearson entre las subescalas del ESQUIZO-Q y las subescalas del SPQ Breve (Raine y Benishay, 1995) y de la Reynolds Adolescent Depression Scale (RADS) (Reynolds, 2002). Los resultados encontrados indicaron elevadas correlaciones entre las dimensiones del ESQUIZO-Q y el SPQ-B, así como entre las subescalas de la RADS y del ESQUIZO$\mathrm{Q}$, apoyando la validez convergente del cuestionario.

\section{Funcionamiento diferencial de los ítems (DIF)}

Una de las principales amenazas contra la validez de las puntuaciones en los cuestionarios proviene de que la probabilidad de respuesta a los ítems no dependa únicamente del nivel del participante en la variable objeto de medición, sino que se encuentre condicionada por la influencia de otras variables ( $p$. ej., sexo o grupo cultural de pertenencia), generando una falta de equivalencia métrica entre las puntuaciones (Elosúa, 2003). En el proceso de construcción y validación del ESQUIZO-Q se llevó a cabo un estudio del funcionamiento diferencial de los ítems (DIF) en función del sexo de los participantes. Los datos indicaron que ninguno de los 51 ítems que componen el ESQUIZO-Q presentó un funcionamiento diferencial en función del sexo de los adolescentes, garantizando la equidad en el proceso de medición.

\section{c) Estimación de la consistentica interna}

Los niveles de consistencia interna para las diez subescalas del ESQUIZO-Q oscilaron entre 0,62, correspondiente a la subescala Falta de Amigos Íntimos, y 0,90, correspondiente a la subescala Experiencias Perceptivas Extrañas. Los niveles de consistencia interna para las dimensiones de segundo orden fueron: Distorsión de la Realidad -0,88-, Anhedonia -0,67- y Desorganización Interpersonal $-0,83$ -

\section{d) Influencia del sexo y la edad en las dimensiones de la personalidad esquizotípica}

El sexo y la edad son dos variables que parecen jugar un rol importante en la expresión del fenotipo psicótico, tanto a nivel clínico como subclínico (American Psychiatric Association, 2000; Goldstein y Link, 1988; Spauwen, Krabbendam, Lieb, Wittchen y van Os, 2003; van Os y Kapur, 2009). En este sentido, si la esquizotipia es considerada como una variante atenuada de la psicosis, y ambas se encuentran conectadas dentro de un continuo psicopatológico de gravedad, es de suponer que las dos se 
hallen afectadas o moduladas por las mismas variables. Cuando se analizó la expresión fenotípica de las experiencias esquizotípicas a través de las subescalas del ESQUIZO-Q, los resultados fueron coincidentes con esta hipótesis. En relación con el sexo, los datos mostraron que las mujeres obtuvieron una mayor puntuación media en comparación con los varones en las subescalas Pensamiento Mágico, Experiencias Perceptivas Extrañas y Falta de Amigos Íntimos; en cambio, los varones puntuaron más elevado que las mujeres en las subescalas Ideación Referencial, Ideación Paranoide y Anhedonia (Social y Física). Cuando se examinaron los datos en función de la edad, se encontraron diferencias estadísticamente significativas en las subescalas Pensamiento y Lenguaje Raros, Anhedonia Física, Falta de Amigos Íntimos y Ansiedad Social Excesiva. En las subescalas Pensamiento y Lenguaje Raros y Falta de Amigos Íntimos el grupo de 18-19 años obtuvo mayores puntuaciones de edad que los grupos de 14, 15 y 16 años, siendo las diferencias estadísticamente significativas. En la subescala Ansiedad Social Excesiva los participantes con 18-19 años obtuvieron mayores puntuaciones que el grupo de 15 años.

\section{e) Versión abreviada del ESQUIZO-Q}

Construir una versión reducida con garantías psicométricas, no sólo permite ahorrar tiempo y esfuerzo en su administración, sino que además facilita su utilización en otros contextos en los que el estudio de la esquizotipia no sea el principal objetivo de evaluación. Para la construcción abreviada del ESQUIZO-Q (ESQUIZO-Q-A) se seleccionaron aquellos ítems de cada una de las diez subescalas del ESQUIZO-Q que presentaban una mayor carga factorial. Esta versión breve finalmente quedó formada por un total de 23 ítems. El estudio de la estructura interna de la versión abreviada, mediante un ACP de segundo orden a nivel de los ítems, también arrojó una solución tridimensional, consistente con la del ESQUIZO-Q, concretada en las dimensiones: Distorsión de la Realidad, Anhedonia y Desorganización Interpersonal. Los niveles de consistencia interna para estas dimensiones fueron respectivamente $0,70,0,67$ y 0,71. Además, el ESQUIZO-Q-A mostró correlaciones moderadas, tanto con el ESQUIZO-Q, como con las subescalas del SPQBreve y de la RADS.

\section{f) ESQUIZO-Q: una herramienta epidemiológica}

Los síntomas psicóticos atenuados -también conocidos como síntomas positivos de la esquizotipia- son un conjunto de experiencias similares a las encontradas en pacientes con esquizofrenia que se manifiestan a nivel subclínico y que no necesariamente se relacionan con una alteración psicológica, médica o de otro tipo. El análisis descriptivo de los ítems del ESQUIZO-Q también permite obtener y analizar el porcentaje de experiencias psicóticas

Tabla 2. Porcentaje de participantes que puntuaron cuatro ("de acuerdo") o cinco ("totalmente de acuerdo") en diez ítems del ESQUIZO-Q: Cuestionario Oviedo para la Evaluación de la Esquizotipia, que miden síntomas psicóticos atenuados

\begin{tabular}{|c|c|c|c|}
\hline Ítems & $\begin{array}{c}\text { Total }(n=1653) \\
n(\%)\end{array}$ & $\begin{array}{c}\text { Varones }(n=794) \\
n(\%)\end{array}$ & $\begin{array}{c}\text { Mujeres }(n=859) \\
n(\%)\end{array}$ \\
\hline $\begin{array}{l}\text { 1."Creo que las cosas que salen en la radio o en la televisión tienen un significado } \\
\text { especial para mí, que mis amigos no entienden" }\end{array}$ & $90(5,5)$ & $54(6,8)$ & $36(4,2)$ \\
\hline 2."Creo que hay gente que puede leer la mente de otras personas" & $177(10,7)$ & $79(9,9)$ & $98(11,4)$ \\
\hline 3."Creo que existen personas que pueden controlar los pensamientos de otras" & $103(6,2)$ & $50(6,3)$ & $53(6,2)$ \\
\hline 4."Estando solo en casa he tenido la sensación de que alguien me hablaba" & $176(10,2)$ & $78(9,8)$ & $98(11,4)$ \\
\hline 5."Oigo voces que los demás no pueden oír" & $54(3,3)$ & $35(4,4)$ & $19(2,2)$ \\
\hline 6."Cuando estoy solo tengo la sensación de que alguien susurra mi nombre" & $62(3,8)$ & $29(3,6)$ & $33(3,8)$ \\
\hline 7."Tengo pensamientos tan reales que parece como si alguien me hablase" & $183(11,1)$ & $92(11,5)$ & $91(10,6)$ \\
\hline 8."Creo que alguien trama algo contra mî" & $159(9,6)$ & $86(10,8)$ & $73(8,5)$ \\
\hline 9."Alguien me la tiene jurada" & $288(17,4)$ & $142(17,9)$ & $146(17)$ \\
\hline 10."Mis compañeros la tienen tomada conmigo" & $44(2,7)$ & $25(3,1)$ & $19(2,2)$ \\
\hline
\end{tabular}

Nota: tomado de Fonseca-Pedrero et al. (2009) 
autoinformadas por los adolescentes. Recientemente, Fonseca-Pedrero et al. (2009) examinaron el número y porcentaje de participantes que marcaron la puntuación 4 ("De acuerdo") o 5 ("Totalmente de acuerdo") en las opciones de respuesta a diez ítems del ESQUIZO-Q (véase Tabla 2). Como puede observarse, entre un 5,5 y un $10,7 \%$ de los adolescentes manifestaron síntomas relacionados con el pensamiento mágico (ítems 1 a 3); entre un 3,3 y un $11,1 \%$ refirieron experiencias perceptivas extrañas (ítems 4 a 7); finalmente, entre un 2,7 y un $17,4 \%$ de los adolescentes refirieron síntomas de ideación paranoide (ítems 8 a 10). Estos resultados muestran, por un lado, que los síntomas psicóticos subclínicos son un fenómeno psicológico bastante común dentro de este grupo de la población y, por otro, que el ESQUIZO-Q es una herramienta interesante para la evaluación de los síntomas psicóticos atenuados en población adolescente.

\section{g) Nuevos datos del ESQUIZO-Q}

Recientemente nuestro equipo de investigación ha realizado nuevos estudios con el ESQUIZO-Q. La finalidad fue replicar en nuevas muestras representativas de la población general adolescente los resultados previos encontrados, así como obtener nuevas evidencias de validez. En un primer estudio, al igual que el llevado a cabo durante el proceso de construcción del ESQUIZO-Q, se realizó un mues- treo estratificado por conglomerados a nivel de aula en el Principado de Asturias. La muestra final la formaron un total de 1438 estudiantes, 693 varones $(48,2 \%)$ y $745(51,9 \%)$ mujeres, pertenecientes a 90 aulas y 28 centros educativos. La media de edad fue de 15,92 años $(D T=1,17)$, oscilando el rango de edad entre los 14 y los 18 años. Los resultados confirmaron de manera fidedigna las 10 subescalas del ESQUIZO-Q, así como la estructura dimensional de tres dimensiones generales de esquizotipia encontrada durante el proceso de construcción de la prueba. Asimismo, las subescalas del ESQUIZO-Q mostraron correlaciones moderadas con las escalas de otros autoinformes como el Personality Diagnostic Questionnaire-4+ (PDQ-4+) (Hyler, 1994) y el Strengths and Difficulties Questionnaire (SDQ) (Goodman, 1997), apoyando nuevamente la validez convergente-discriminante del cuestionario. Las correlaciones entre las subescalas del PDQ-4+ y las dimensiones generales del ESQUIZO-Q se recogen en la Tabla 3.

En un segundo estudio, utilizando una muestra de 537 adolescentes, se examinó la relación entre las puntuaciones del ESQUIZO-Q y el reconocimiento facial de emociones. La capacidad para reconocer rostros se encuentra con frecuencia alterada en pacientes con esquizofrenia y, además, ha demostrado ser un buen indicador de riesgo de psicosis (Brown y Cohen, 2010). Para la evaluación del reconocimiento facial se presentaban en un ordenador fotografías que expresaban las 6 emociones básicas

Tabla 3. Correlaciones de Pearson entre las subescalas del Personality Diagnostic Questionnaire-4+ (PDQ-4+) y las dimensiones del ESQUIZO-Q: Cuestionario Oviedo para la Evaluación de la Esquizotipia

ESQUIZO-Q

\begin{tabular}{lccc}
\hline \multicolumn{1}{c}{ PDQ-4+ } & Anhedonia & Distorsión de la Realidad & Desorganización Social \\
\hline Paranoide &,$- 09^{*}$ &, $58^{*}$ &, $59^{*}$ \\
Esquizoide &, $19^{*}$ &, $33^{*}$ &, $43^{*}$ \\
Esquizotípica &, 03 &, $72^{*}$ &, $69^{*}$ \\
Antisocial &, $08^{*}$ &, $42^{*}$ &, $35^{*}$ \\
Borderline &,$- 07^{*}$ &, $61^{*}$ &, $64^{*}$ \\
Histriónica &,$- 11^{*}$ &, $44^{*}$ &, $37^{*}$ \\
Narcisista &,- 03 &, $51^{*}$ &, $46^{*}$ \\
Obsesivo-Compulsivo &,$- 18^{*}$ &, $41^{*}$ &, $47^{*}$ \\
Evitativa &,$- 07^{*}$ &, $38^{*}$ &, $63^{*}$ \\
Dependiente &,- 03 &, $43^{*}$ &, $55^{*}$ \\
\hline p & & & \\
\hline
\end{tabular}


(asco, ira, alegría, tristeza, miedo y sorpresa) en un grado de intensidad variable. En la parte inferior de la fotografía se presentaban un total de 8 palabras que se correspondían a dichas emociones básicas, y a 2 emociones sociales. La tarea de los alumnos consistió en indicar cuál era la emoción que se correspondía con la expresada en la fotografía. Los resultados mostraron que los participantes con puntuaciones elevadas en las dimensiones del ESQUIZO$Q$ presentaban una mayor tasa de errores en el reconocimiento facial de emociones en comparación con aquellos que presentaron puntuaciones bajas. Estos datos son convergentes con los estudios llevados a cabo en pacientes con esquizofrenia e informan de un déficit en los procesos controlados de reconocimiento emocional en este conjunto de participantes con una mayor vulnerabilidad teórica a la esquizofrenia.

\section{Recapitulando}

El ESQUIZO-Q (Fonseca-Pedrero, Muñiz et al., 2010) presenta un comportamiento psicométrico adecuado que permite la evaluación de los rasgos esquizotípicos de la personalidad -considerados éstos como un marcador de riesgo para la esquizofrenia- de forma rápida, sencilla y no invasiva. Este autoinforme puede ser utilizado como método de cribado psicopatológico en población adolescente en una amplia variedad de contextos (clínicos, educativos y de investigación), con la finalidad de seleccionar a participantes "de riesgo" y llevar a cabo una evaluación psicológica más exhaustiva. Asimismo, el ESQUIZO-Q es una herramienta de evaluación que puede ser empleada con fines epidemiológicos en el estudio de las experiencias psicóticas subclínicas en muestras comunitarias de adolescentes (Fonseca-Pedrero, Lemos-Giráldez, Paino, Sierra-Baigrie et al., 2009).

En primer lugar, el ESQUIZO-Q se construyó a partir de una revisión exhaustiva de la literatura previa (Fonseca-Pedrero et al., 2008) y siguiendo las directrices para el desarrollo y traducción de tests y construcción de ítems. En segundo lugar, se incorporó un formato de respuesta tipo Likert que, como señala la literatura psicométrica al respecto, mejora la fiabilidad y la validez de las puntuaciones, así como la satisfacción de respuesta de los usuarios (Muñiz, García-Cueto y Lozano, 2005). En tercer lugar, el ESQUIZO-Q es un autoinforme sencillo compuesto por un total de 51 ítems que se aplica de forma rápida y no invasiva, lo que influye en el ahoro de tiempo y la motivación de los participantes. En cuarto lugar, los niveles de consistencia interna para las subescalas del ESQUIZO-Q oscilaron entre 0,62 y 0,90 . En quinto lugar, el análisis del funcionamiento diferencial de los ítems (DIF) permitió eliminar aquellos ítems cuya probabilidad de respuesta difería en función del sexo de los adolescentes. El DIF ha sido escasamente investigado dentro de esta área de estudio, siendo un aspecto muy importante en la validez de los datos (American Educational Research Association et al., 1999). En sexto lugar, el análisis de la estructura interna del ESQUIZO-Q respaldó empíricamente las 10 subescalas propuestas por los autores. Las subescalas se derivaron utilizando una estrategia de reducción de ítems empírica, de acuerdo a criterios psicométricos debidamente explicitados. Del mismo modo, y en consonancia con la literatura previa, la estructura de la esquizotipia, medida a través del ESQUIZO-Q, parece ser un constructo de naturaleza multidimensional (Fonseca-Pedrero, Lemos-Giráldez, Paino, Villazón-García et al., 2009; Fonseca-Pedrero, Paino et al., 2009; Raine, 2006). El análisis de validez de constructo, realizado a nivel de los ítems, reveló la presencia de tres componentes de segundo orden o dimensiones generales de esquizotipia, a saber: Distorsión de la Realidad, Anhedonia y Desorganización Interpersonal. Estos tres componentes fueron totalmente coincidentes con el análisis de la estructura interna realizado con otra muestra representativa de la población general adolescente, por lo que los datos obtenidos respaldan la subescalas del ESQUIZO-Q propuestas en su proceso inicial de construcción y validación. En séptimo lugar, se encontró una adecuada validez convergente-discriminante entre las subescalas del ESQUIZO-Q y las subescalas del SPQ-B, de la RADS, del SDQ y del PDQ-4+. En octavo lugar, se dispone de una versión abreviada del ESQUIZO-Q que permite su utilización en investigaciones o en contextos aplicados donde el objetivo principal no sea recoger informa- 
ción de la esquizotipia, pero sí resulte interesante indagar sobre ella y su relación con otros constructos.

Los jóvenes encuestados con este tipo de autoinformes (Fonseca-Pedrero et al., 2008) podrían ser considerados como pseudo-esquizotípicos desde el Modelo biopsicosocial propuesto por Raine (2006). Este modelo postula la existencia de dos tipos de esquizotipia con etiologías diferentes, a saber: la neuro-esquizotipia y la pseudo-esquizotipia. La diferenciación entre ambas sería una cuestión más bien relativa que absoluta (véase Tabla 4). La neuroesquizotipia tendría su origen predominantemente, aunque no exclusivamente, en procesos genéticos, del neurodesarrollo y neurobiológicos; presentaría fundamentalmente rasgos de tipo interpersonal y de desorganización cognitiva, no estaría asociada con adversidad ambiental, presentaría una mayor estabilidad temporal, estaría más indicado un tratamiento de tipo psicofarmacológico y sería un claro marcador de riesgo para la esquizofrenia. En cambio, en la pseudo-esquizotipia, las influencias ambientales tendrían un papel más preponderante, tanto las ocurridas durante el periodo postnatal como las influencias de tipo psicosocial (p. ej., abuso sexual, discriminación o problemas de relación); predominarían los rasgos de tipo cognitivo-perceptual, no necesariamente sería un factor de riesgo para la esquizofrenia, mostraría una mayor fluctuación temporal y respondería mejor a tratamientos de tipo psicológico.

Finalmente, se debe mencionar que en aquellos adolescentes que obtienen altas puntuaciones en el ESQUIZO-Q sería necesario, como requisito imprescindible, llevar a cabo una evaluación psico- lógica más pormenorizada, que podría incluir la aplicación de otro tipo de instrumentos; a saber, cuestionarios de funcionamiento social, entrevistas al alumno y a la familia, o incluso pruebas de laboratorio que exploren funciones neuropsicológicas. La utilización combinada de varios paradigmas de investigación permite, por un lado, mejorar la capacidad de detectar individuos de riesgo a la esquizofrenia, y por otro, comprender y analizar la esquizofrenia, así como su detección e intervención dentro de los modelos biopsicosociales, teniendo en cuenta la naturaleza multicausal de este cuadro psicopatológico.

Futuros estudios deberían seguir profundizando en la calidad métrica del ESQUIZO-Q, particularmente en lo relacionado con su capacidad predictiva (continuidad temporal), así como en su utilización combinada con otros paradigmas de investigación; por ejemplo, su implementación en hijos de pacientes con esquizofrenia (continuidad genética), de cara a aumentar su valor predictivo en la detección de participantes de riesgo de psicosis. No obstante, y como no puede ser de otro modo, es necesario que este tipo de investigaciones y resultados se hagan eco en otros sectores de la población y que adquieran un sentido práctico. Futuras iniciativas que se lleven a cabo en la detección temprana de los problemas psicológicos graves como la esquizofrenia, deberían facilitar a los centros escolares, sanitarios y a las familias el acceso a este tipo de herramientas, trabajando en el desarrollo de programas específicos de formación (p. ej., sobre la detección de posibles señales de alerta o de riesgo). Destacar además que la identificación temprana de individuos de riesgo pasa por la participación, involucración y coordina-

Tabla 4. Modelo Biopsicosocial de Raine (2006)

\begin{tabular}{|c|c|c|}
\hline Variable & Neuro-esquizotipia & Pseudo-esquizotipa \\
\hline \multirow[t]{2}{*}{ Origen preferentemente } & Genético & Psicosocial \\
\hline & Historia familiar & Adversidad psicosocial \\
\hline Inicio & Temprano & Temprano o tardío \\
\hline Rasgos predominantes & Interpersonal-Desorganizado & Cognitivo-Perceptual \\
\hline Tratamiento preferente & Psicofarmacológico & Psicológico \\
\hline Adversidades psicológicas & No necesariamente & $\mathrm{Si}$, postnatal y psicosocial \\
\hline Riesgo para la esquizofrenia & $\mathrm{Si}$ & No \\
\hline Identificación a través de & Marcadores neuroevolutivos & Autoinformes \\
\hline Fluctuación en el tiempo & Estable & Inestable \\
\hline
\end{tabular}


ción directa de todos los sectores de la comunidad educativa (orientadores, profesores, padres, etc.), así como de otros profesionales (psicólogo, pedagogos, médicos, enfermeros, trabajadores sociales). No se debe perder de vista que la finalidad última no es otra que mitigar el posible impacto que pueda ocasionar la esquizofrenia en las esferas personal, familiar, académica y social de las personas.

\section{Extended Summary}

Schizophrenia is a chronic, severe, and disabling mental disorder which has a direct impact on the personal, familial and social spheres of the individual, and has its onset in late adolescence or early adulthood. Clinical evidence suggests that a prompt detection of the psychotic state with an effective posterior early intervention, not only improves disorder prognosis in the short, medium and longterms, but also improves the results of treatment. Moreover, in the case of apparition of the disorder, recovery is much faster, decreasing the time of hospitalization and the associated negative consequences (e.g., comorbidity) and the impact caused by the disorder. Therefore, the prevention and early detection of this disorder is one of the main priorities of mental health services.

The aim of the "psychometric high-risk" paradigm is the detection, by means of self-report measures and based on score profiles, of those individuals with a higher theoretical vulnerability of transiting toward a psychotic disorder in the future. There are over a dozen longitudinal studies indicating that participants with high scores on these types of self-reports have an increased probability of transiting toward a severe psychological disorder as well as of presenting several affective, cognitive, behavioral, neuropsychological and neurodevelopmental alterations which show the "at risk mental state" of these types of individuals. There is a wide variety of instruments in the literature for the assessment of psychosis proneness in the general population, however, to date there was no instrument specifically designed for the assessment of schizotypal traits in Spanish adolescents and that incorporates the advances developed in education and psychological assessment in the last few years. This article presents data relative to the construction and validation of the ESQUIZO-Q "Cuestionario Oviedo para la Evaluación de la Esquizotipia” (The Oviedo Schizotypy Assessment Questionnaire) in a representative sample of nonclinical adolescents. The availability of brief, simple and easily administered screening instruments for the identification of participants who are at risk of developing a schizophrenia-spectrum disorder, with a view to conducting a more exhaustive assessment, is of vital importance from both clinical and research points of view. The results indicate that the ESQUIZO-Q is a self-report with adequate psychometric properties for the assessment of schizotypal traits in Spanish adolescent populations. First, the ESQUIZO-Q was constructed based on an exhaustive review of the previous literature and following the guidelines for the development and translation of tests and item construction. Second, the ESQUIZO$\mathrm{Q}$ is a simple self-report composed of 51 items that is applied in a rapid and noninvasive manner, which saves time and improves participant motivation. Third, the levels of internal consistency found for ESQUIZO-Q subscales ranged from .62 to .90 . Fourth, the analysis of the differential item functioning permitted the elimination of those items whose probability of response varied according to gender of the adolescents. This aspect endorses the validity of the data. Fifth, the analysis of the internal structure of the ESQUIZO-Q offered empirical support to the 10 subscales proposed by the authors. The analysis of construct validity, conducted at the item level, revealed the presence of three second-order components or general dimensions of schizotypy, namely: Distortion of Reality, Anhedonia and Interpersonal Disorganiza-tion. Sixth, an adequate convergent-discriminant validity was found between the ESQUIZO-Q subscales and the subscales of different self-reports that assessed depressive, emotional and behavioral symtomatology and personality-disorder traits. Seventh, an abbreviated version of the ESQUIZO-Q is available that can be used in research or applied contexts where the main objective is not to gather information regarding schizotypy but it is interesting to investigate its relation to other constructs. Eight, the ESQUIZO-Q is an assessment tool that can be used for epidemiological purposes in the 
study of subclinical psychotic experiences in community samples of adolescents.

This self-report can be utilized as a psychopathological screening method in adolescent populations in a wide variety of settings (clinical, educational and research) with the aim of selecting the "at risk" participants and conducting a more exhaustive psychological assessment which could include the application of other types of instruments; namely, social functioning questionnaires, interviews with the adolescent and family, or even laboratory tests exploring neuropsychological functions (e.g., sustained attention). Nevertheless, and as it could be no other way, it is necessary for this type of investigation and results to be known in other sectors of the population in order to acquire a practical sense. The combined use of several research paradigms (e.g., studies of patients' first-order relatives) allows, on the one hand, the improvement of the predictive capacity of individuals at risk for schizophrenia, and on the other hand, the comprehension and analysis of schizophrenia as well as its detection and intervention within biopsychosocial models taking into consideration the multicausal nature of this psychopathological disorder.

Future initiatives for the early detection of severe psychological problems should offer educational and health centres and families the possibility of using these types of tools, working on the development of specific training programs (e.g., regarding the detection of possible alert or risk signs). It should also be highlighted that the early identification of at risk individuals requires the participation, implication and direct coordination of all the sectors of the education community (guidance counselors, teachers, parents, etc.) as well as other professionals (pedagogues, doctors, nurses, social workers). It should be kept in mind that the final aim is to mitigate the possible impact that schizophrenia may have on the personal, familial, academic and social spheres of individuals.

\section{Referencias}

American Educational Research Association, American Psychological Association y National
Council on Measurement in Education. (1999). Standars for Educational and Psychological Testing. Washington, D.C.: Author.

American Psychiatric Association. (2000). Diagnostic and Statistical Manual of Mental Disorders (4th ed revised) Washington, DC: American Psychiatric Association.

Barrantes Vidal, N., Ros-Morente, A. y Kwapil, T. R. (2009). An examination of neuroticism as a moderating factor in the association of positive and negative schizotypy with psychopathology in a nonclinical sample. Schizophrenia Research, 115, 303-309.

Brown, C. y Faraone, S. (2004). Prevención de la esquizofrenia y de la conducta psicótica: definiciones y aspectos metodológicos. En W. S. Stone, S. Faraone y M. T. Tsuang (Eds.), Intervención clínica precoz y prevención de la esquizofrenia (pp. 255-284). Barcelona: J \& C ediciones.

Brown, L. A. y Cohen, A. S. (2010). Facial emotion recognition in schizotypy: the role of accuracy and social cognitive bias. Journal of the International Neuropsychological Society, 16, 474-483.

Cyhlarova, E. y Claridge, G. (2005). Development of a version of the Schizotypy Traits Questionnaire (STA) for screening children. Schizophrenia Research, 80, 253-261.

Chapman, J. P., Chapman, L. J. y Kwapil, T. R. (1995). Scales for the measurement of schizotypy. En A. Raine, T. Lencz y S. A. Mednick (Eds.), Schizotypal Personality (pp. 79-106). New York: Cambridge University Press.

Chapman, J. P., Chapman, L. J., Raulin, M. L. y Eckblad, M. (1994). Putatively psychosis-prone subjects 10 years later. Journal of Abnormal Psychology, 87, 399-407.

DiDuca, D. y Joseph, S. (1999). Assessing schizotypal traits in 13-18 year olds: Revising the JSS. Personality and Individual Differences, 27, 673682.

Dominguez, M. G., Wichers, M., Lieb, R., Wittchen, H.-U. y van Os, J. (in press). Evidence that onset of clinical psychosis is an outcome of progressively more persistent subclinical psychotic experiences: An 8-Year Cohort Study. Schizophrenia Bulletin. 
Downing, S. M. (2006). Twelve steps for effective test development. En S. M. Downing y T. M. Haladyna (Eds.), Handbook of test development (pp. 3-25). Mahwah, NJ: Lawrence Erlbaum Associates.

Elosúa, P. (2003). Sobre la validez de los tests. Psicothema, 15, 315-321.

Fonseca-Pedrero, E., Lemos-Giráldez, S., PaínoPiñeiro, M., Villazón-García, U. y Muñiz, J. (2010). Schizotypal traits, obsessive-compulsive symptoms, and social functioning in adolescents. Comprehensive Psychiatry, 51, 71-77.

Fonseca-Pedrero, E., Lemos-Giráldez, S., Paino, M., Sierra-Baigrie, S., Villazón-García, U., GarcíaPortilla, M. y Muñiz, J. (2010). Dimensionality of hallucinatory predisposition: Confirmatory factor analysis of the Launay-Slade Hallucination Scalerevised in college students. Anales de Psicología, 26, 41-48.

Fonseca-Pedrero, E., Lemos-Giráldez, S., Paino, M., Sierra-Baigrie, S., Villazón-García, U. y Muñiz, J. (2009). Experiencias psicóticas atenuadas en población adolescente [Attenuated psychotic experiences in adolescents]. Papeles del Psicólogo, 30, 63-73.

Fonseca-Pedrero, E., Lemos-Giráldez, S., Paino, M., Villazón-García, U. y Muñiz, J. (2009). Validation of the Schizotypal Personality Questionnaire Brief form in adolescents. Schizophrenia Research, 111, 53-60.

Fonseca-Pedrero, E., Linscott, R. J., LemosGiráldez, S., Paino, M. y Muñiz, J. (in press). Psychometric properties of two measures for the assessment of schizotypy in adolescents. Psychiatry Research, doi: 10.1016/j.psychres. 2009.1002.1012.

Fonseca-Pedrero, E., Muñiz, J., Lemos-Giráldez, S., Paino, M. y Villazón-García, U. (2010). ESQUIZO-Q: Cuestionario Oviedo para la Evaluación de la Esquizotipia [ESQUIZO-Q: Oviedo Questionnaire for Schizotypy Assessment ]. Madrid: TEA ediciones.

Fonseca-Pedrero, E., Paino, M., Lemos-Giráldez, S., García-Cueto, E., Campillo-Álvarez, A., Villazón-García, U. y Muñiz, J. (2008). Schizotypy assessment: State of the art and future prospects. International Journal of Clinical and
Health Psychology, 8, 577-593.

Fonseca-Pedrero, E., Paino, M., Lemos-Giráldez, S., Villazón-García, U., García-Cueto, E., Bobes, J. y Muñiz, J. (2009). Versión reducida del Cuestionario TPSQ de Estilos Perceptuales y de Pensamiento. Psicothema, 21, 499-505.

Goldstein, J. M. y Link, B. G. (1988). Gender and the expression of schizophrenia. Journal of Psychiatric Research, 22(2), 141-155.

Gooding, D. C., Tallent, K. A. y Matts, C. W. (2005). Clinical status of at-risk individuals 5 years later: Further validation of the psychometric high-risk strategy. Journal of Abnormal Psychology, 114, 170-175.

Goodman, R. (1997). The strengths and difficulties questionnaire: a research note. Journal of Child Psychology and Psychiatry, 38, 581-586.

Hyler, S. E. (1994). The Personality Diagnostic Questionnaire 4+. New York: New York State Psychiatric Institute.

Johnstone, E. C., Ebmeier, K. P., Miller, P., Owen, D. G. C. y Lawrie, S. M. (2005). Predicting schizophrenia: findings from the Edinburgh High-Risk Study. British Journal of Psychiatry, 186, 18-25.

Kwapil, T. R. (1998). Social anhedonia as a predictor of the development of schizophrenia-spectrum disorders. Journal of Abnormal Psychology, 107(4), 558-565.

Kwapil, T. R., Barrantes Vidal, N. y Silvia, P. J. (2008). The dimensional structure of the Wisconsin schizotypy scales: Factor identification and construct validity. Schizophrenia Bulletin, 34, 444-457.

Lemos-Giráldez, S., Vallina-Fernández, O., Fernández-Iglesias, P., Vallejo-Seco. G., FonsecaPedrero, E., Paíno-Piñeiro, M., Sierra-Baigrie, S., García-Pelayo, P., Pedrejón-Molino, C., AlonsoBada, S., Gutiérrez-Pérez, A. y Ortega-Ferrández, J. A. (2009). Symptomatic and functional outcome in youth at ultra-high risk for psychosis: A longitudinal study. Schizophrenia Research, 115, 121-129.

Lenzenweger, M. F. (1994). Psychometric high-risk paradigm, perceptual aberrations, and schizotypy: An update. Schizophrenia Bulletin, 20, 121-135.

Lenzenweger, M. F., McLachlan, G. y Rubin, D. B. (2007). Resolving the latent structure of schizo- 
phrenia endophenotypes using expectation-maximization-based finite mixture modeling. Journal of Abnormal Psychology, 116(1), 16-29.

Mason, O. y Claridge, G. (2006). The OxfordLiverpool Inventory of Feelings and Experiences (O-LIFE): Further description and extended norms. Schizophrenia Research, 82(2), 203-211.

McGlashan, T. H. y Johannessen, J. O. (1996). Early detection and intervention whith schizophrenia: research. Schizophrenia Bulletin, 22, 201-222.

McGorry, P. D., Krstev, H. y Harrigan, S. (2000). Early detection and treatment delay: implications for outcome in early detection. Current Opinion Psychiatry, 13, 37-43.

Meehl, P. E. (1962). Schizotaxia, schizotypy, schizophrenia. American Psychologist, 17, 827-838.

Moreno, R., Martínez, R. y Muñiz, J. (2006). New guidelines for developing multiple-choice items. Methodology, 2, 65-72.

Moreno, R., Martínez, R. J. y Muñiz, J. (2004). Directrices para la construcción de ítems de elección múltiple. Psicothema, 16, 490-497.

Morrison, A. P., French, P., Lewis, S., Roberts, M., Raja, S., Neil, S., Parker, S., Green, J., Kilcommons, A., Walford, L. y Bentall, R. (2006). Psychological factors in people at ultra-high risk of psychosis: Comparison with non-patients and associations with symptoms. Psychological Medicine, 36, 1395-1404.

Muñiz, J., Fidalgo, A. M., García-Cueto, E., Martínez, R. y Moreno, R. (2005). Análisis de los items. Madrid: La Muralla.

Muñiz, J. y Fonseca-Pedrero, E. (2008). Construcción de instrumentos de medida para la evaluación universitaria. Revista de Investigación en Educación, 5, 13-25.

Muñiz, J., García-Cueto, E. y Lozano, L. M. (2005). Item format and the psychometric properties of the Eysenck Personality Questionnaire. Personality and Individual Differences, 38, 61-69.

Obiols, J. E., Barragán, M., Vicens, J. y Navarro, J. B. (2008). Síntomas seudo-psicóticos en adolescentes de la población general. Revista de Psicopatología y Psicología Clínica, 13, 205217.

Poulton, R., Caspi, A., Moffitt, T. E., Cannon, M., Murray, R. y Harrington, H. (2000). Children's self-reported psychotic symptoms and adult schizophreniform disorder: a 15-year longitudinal study. Archives of General Psychiatry, 57, 10531058.

Raine, A. (1991). The SPQ: A scale for the assessment of schizotypal personality based on DSM-III$\mathrm{R}$ criteria. Schizophrenia Bulletin, 17, 555-564.

Raine, A. (2006). Schizotypal personality: Neurodevelopmental and psychosocial trajectories. Annual Review of Clinical Psychology, 2, 291-326.

Raine, A. y Benishay, D. (1995). The SPQ-B: A brief screening instrument for schizotypal personality disorder. Journal of Personality Disorders, 9, 346-355.

Rawlings, D. y MacFarlane, C. (1994). A multidimensional schizotypal traits questionnaire for young adolescents. Personality and Individual Differences, 17(4), 489-496.

Reynolds, W. M. (2002). Reynolds Adolescent Depression Scale-2nd Edition. Professional manual. Odessa: Psychological Assessment Resources, Inc.

Spauwen, J., Krabbendam, L., Lieb, R., Wittchen, H.U. y van Os, J. (2003). Sex differences in psychosis: normal or pathological? Schizophrenia Research, 62, 45-49.

Vallina, O., Lemos Giráldez, S. y Fernández, P. (2006). Estado actual de la detección e intervención temprana en psicosis. Apuntes de Psicología, $24,185-221$.

van Os, J. y Kapur, S. (2009). Schizophrenia. Lancet, 374, 635-645.

van Os, J., Linscott, R. J., Myin-Germeys, I., Delespaul, P. y Krabbendam, L. (2009). A systematic review and meta-analysis of the psychosis continuum: Evidence for a psychosis pronenesspersistence-impairment model of psychotic disorder. Psychological Medicine, 39, 179-195.

Welham, J., Scott, J., Williams, G., Najman, J., Bor, W., O'Callaghan, M. y McGrath, J. (2009). Emotional and behavioural antecedents of young adults who screen positive for non-affective psychosis: a 21-year birth cohort study. Psychological Medicine, 39, 625-634.

Yung, A., Philips, L. J., Pan Yuen, H. y McGorry, P. D. (2004). Risk factors for psychosis in an 
ultra high-risk group: Psychopathology and clinical features. Schizophrenia Research, 67, 131-142.

Yung, A. R., Killackey, E., Hetrick, S. E., Parker, A.
G., Schultze-Lutter, F., Klosterkoetter, J., Purcell, R. y Mcgorry, P. D. (2007). The prevention of schizophrenia. International Review of Psychiatry, 19, 633-646.

Manuscrito recibido: $17 / 08 / 2010$

Revisión eecibida: 28/09/2010

Manuscrito aceptado: 04/10/2010 